http://doi.org/10.15359/ree.12-Ext.1

\title{
ALGUNOS AVANCES Y PROYECCIONES EN EL CAMPO DE LA PEDAGOGÍA RURAL
}

\author{
Alicia Díaz Alvarado \\ Académica de la División de Educación Rural. CIDE-Universidad Nacional \\ Heredia, Costa Rica
}

Recibido 18 de junio 2007 • Aprobado 15 de octubre 2007

\begin{abstract}
Resumen: Este artículo explora la forma en que se ha hecho Pedagogía Rural en la División de Educación Rural (DER) del Centro de Investigación y Docencia en Educación (CIDE), de la Universidad Nacional. Para destacar los avances de los últimos tiempos, se organiza este accionar pedagógico en dos grandes momentos, cada uno de ellos con sus características, logros y debilidades. Aunque describe con cierto detalle la forma en que se ha hecho Pedagogía Rural en el camino recorrido por la División, lo importante es destacar cómo esto permite un salto cualitativo hacia nuevos horizontes en la Pedagogía Rural que hace la DER. El artículo finaliza con algunas reflexiones sobre los factores que posiblemente decidirán el destino de la Pedagogía Rural en los ámbitos nacional y centroamericano.
\end{abstract}

Palabras clave: Pedagogía, Pedagogía Rural, formación de educadores rurales.

\begin{abstract}
This article explores how Rural Pedagogy has been developed within the "División de Educación Rural (DER)" of the "Centro de Investigación y Docencia en Educación (CIDE)" at the "Universidad Nacional". In order to emphasize recent advancements, pedagogical activities are organized in two different moments, taking into account its characteristics, strengths and weaknesses. Even though, the way rural pedagogy has been done at Division's history is described in detail, the main interest is to accentuate how this allows a quantum shift towards new horizons in rural pedagogy inside the DER. The article concludes providing some thoughts concerning the factors that will probably decide the destiny of Rural Pedagogy nationally and in Central America.
\end{abstract}

Key words: Pedagogy, Rural Pedagogy, Rural Education Training.

\section{INTRODUCCIÓN}

Hace aproximadamente veinticinco años se escribió, en Costa Rica, un primer documento sobre Pedagogía Rural, producto de un proceso reflexivo sobre la teoría y la práctica de procesos educativos desarrollados en zonas rurales, según fue experimentado por el equipo profesional que

\footnotetext{
Dra. Académica de la División de Educación Rural, Centro de Investigación y Docencia en Educación (CIDE), Universidad Nacional en Costa Rica. Forma parte del equipo de profesores de la Maestría en Educación Rural Centroamericana de esta Universidad. Dirección electrónica: Correo electrónico: adiaz@una.ac.cr
} 
asumió este objeto de estudio en la Universidad Nacional, Costa Rica. El título de este documento es Hacia una Pedagogía Rural (Aguilar y Monge, 1994). título muy pertinente, pues refleja incomplitud, proceso, avances y construcción paulatina; de esa manera, el texto incentivó para continuar esfuerzos por profundizar en el tema.

Aunque es justo reconocer que en Costa Rica se hacía Educación Rural desde mucho tiempo, desde el punto de vista de la sistematización de conocimientos, este período constituyó un primer momento en el desarrollo de esta subdisciplina en Costa Rica.

Uno de los méritos más importantes de este trabajo, consiste en haber recogido la experiencia desarrollada por un grupo de profesionales que se especializaron en atender la educación en la ruralidad, desde la educación superior.

El trabajo de práctica-teoría y teoría-práctica continuó, dando así lugar a nuevos rumbos en lo que respecta a este tema. Hay que tomar en cuenta que todo cambia, y esta secuencia de transformaciones es muy importante en sus consecuencias, porque cambia todo, incluyendo la Pedagogía Rural.

En esta perspectiva, no debe despreciarse el papel que ha tenido el contexto, tanto en el ámbito nacional como internacional, lo mismo que las tendencias políticas, económicas y educativas de la época actual, pues todo se ha conjugado para mostrar un panorama diferente al que reinaba en el primer período; por ello, amerita que destaquemos aquí un segundo momento.

Este trabajo resume los aprendizajes del primer momento, explora el segundo, e intenta algunos descubrimientos del período actual. Finalmente, proyecta la pedagogía rural hacia los retos que se perfilan en el horizonte, los cuales todavía no están claramente definidos.

Antes de entrar en los contenidos mencionados, es importante recordar el concepto de Pedagogía, tal y como se ha conceptualizado en la División de Educación Rural (DER). En este marco, se entiende la Pedagogía como la ciencia que estudia los procesos educativos. Su objeto de estudio es la Educación y, por lo tanto, le corresponde reflexionar, crear teoría y hacer aportes sobre la práctica educativa. También es importante aclarar lo que hemos entendido por Educación; esta se ha conceptualizado como una actividad práctica que tiene que ver con el hecho educativo (Aguilar y Monge, 1994).

Lo anterior nos lleva a entender la Pedagogía rural como el estudio de la situación de la educación en las zonas rurales. En la DER, se hace Pedagogía Rural, pues su objeto de estudio es la Educación Rural. Pero además, se trabaja la Pedagogía Rural desde la práctica, en la medida en que las y los estudiantes son de zonas rurales y laboran en estas regiones. Su realidad es la realidad rural, y por ende, la realidad de referencia de la División es la realidad rural.

\section{HACIENDO PEDAGOGÍA RURAL A PARTIR DE LOS APRENDIZAJES DEL PRIMER MOMENTO}

La idea principal del primer momento consiste en que los procesos educativos que se lleven a cabo en espacios rurales deben ajustarse a las necesidades de la realidad rural. Esta afirmación se convierte en una máxima que debe cumplirse tanto para los procesos educativos institucionalizados, como para aquellos que no lo son. Se aplica este principio a los diferentes procesos que tienen lugar en la educación rural: la escuela rural, actividades con las niñas y niños, acciones con las y los docentes rurales, formación de educadoras y educadores para el área rural y procesos comunitarios, entre otros. Esto implica que la búsqueda de las necesidades es clave, porque en función de ellas se desarrollará el resto. 
El conocimiento de las zonas rurales se hace, entonces, imprescindible. Este conocimiento define la pertinencia de la pedagogía, el valor y el sentido que tenga para los participantes. Además, las características de las zonas rurales cambian constantemente por la dinámica social de los pueblos, y por el efecto mariposa ${ }^{2}$, lo que hace que la tarea de conocer la realidad rural nunca termine. Debe haber un conocimiento base de esta realidad y seguimiento continuo para asegurar actualidad en la información.

Asimismo, es importante considerar que los datos obedezcan no sólo a los aspectos macro; es decir, indicadores poblacionales, socioeconómicos y productivos, tales como el número de habitantes de una comunidad, su condición económica, la tenencia de la tierra, la actividad económica predominante, la tasa de empleo y desempleo, entre otros. Además de estos datos, debe contarse con información micro, relacionada con las motivaciones de las personas, sus anhelos, sus temores, su idiosincrasia, sus creencias, y otros elementos culturales que también definen el comportamiento de una comunidad.

En el conocimiento de las zonas rurales debe contemplarse la escuela rural, entendida como el espacio físico y social en el que se llevan a cabo los procesos educativos en las áreas rurales: su misión, su cobertura, su impacto en el desarrollo, sus características y sus necesidades. La escuela debe conceptualizarse en un sentido amplio, que considere lo físico, lo educativo y lo relativo a las personas involucradas en estos procesos (toda la comunidad). Cabe aclarar que en este contexto, el concepto de escuela no se circunscribe a una planta física, ni tampoco a lo que conocemos como escuela primaria, sino que involucra procesos educativos para todas las edades, en todos los niveles (primaria, secundaria, universitaria), en todas las modalidades existentes (por ejemplo, las diversas opciones para estudiantes rezagados) y en sus diferentes tipos, según la institucionalización de los procesos (educación formal y no formal).

Un aspecto por considerar en el tema del conocimiento de una realidad es qué tanto han participado quienes verdaderamente la conocen; es decir, sus habitantes. Si un diagnóstico de una comunidad se elabora desde afuera, corre el riesgo de ser impreciso, y esto lo invalida. Por ello, la participación de las personas de la comunidad como agentes sociales, es imprescindible.

¿Para qué todo este conocimiento? No tendría sentido contar con éste si no se utiliza. La información debe servir para ajustar los procesos educativos a la realidad rural, y con ello, asegurar pertinencia. Aquí interviene la creatividad del ser humano al determinar cuáles medidas tomar, para asegurar esa pertinencia; en otras palabras, cómo ajustar los procesos educativos al contexto que de previo conocemos.

El currículo se considera en este sentido, no sólo como una disciplina científica que ha logrado acumular teorías, posiciones y procesos, sino también como vivencia, es decir, como una práctica que se ve afectada por las condiciones de la realidad. El cúmulo de experiencias educativas que conforman el currículo en la educación rural debe tener, como punto de referencia fundamental, la realidad rural en sus aspectos macro y micro.

A partir de los aprendizajes mencionados antes, la DER plantea un plan de estudios para docentes de primaria en servicio en zonas rurales, dirigido a maestras y maestros que ejercían esta labor sin título, plan que estuvo ajustado a sus necesidades, en los siguientes aspectos:

- Contenidos: el plan contemplaba el estudio del planeamiento, metodologías para diferentes materias, tecnología educativa, problemas de aprendizaje y otros aspectos de aplicación en las escuelas.

El "efecto mariposa" consiste en pensar que todas las cosas, por pequeñas que parezcan, influyen en todas las demás. A manera de ejemplo se indica que el movimiento de las alas de las mariposas puede generar cambios importantes en el clima, a raíz de la cadena de influencias y de cambios que produce. 
- Periodización: Cursos de verano, que eran teóricos e intensivos y se impartían durante las vacaciones escolares, y cursos de "seguimiento", de los cuales hacían en dos fases, la presencial (interacción con el grupo y el profesor una vez al mes, en fin de semana) y la de distancia (trabajo diario de docente-estudiante, como parte del currículo formativo).

- Itinerancia: Los profesores viajaban a las zonas rurales para impartir lecciones y realizar supervisiones al trabajo de los y las estudiantes. Estos profesores debían cargar, desde Heredia, el material para cada estudiante.

- Proyectos comunitarios: Los estudiantes debían realizar proyectos comunales en los que se atienda una necesidad sentida en la comunidad o en la escuela rural.

Estas medidas tan creativas respondían a las características de la realidad rural de ese momento: los docentes no estaban titulados, el Ministerio de Educación Pública (MEP), sentía la necesidad de profesionalizarlos; estos docentes tenían muchas carencias de conocimiento y simultáneamente, una gran experiencia; las escuelas contaban con muy pocos recursos, estaban ubicadas en lugares muy lejanos y el calendario escolar iba de marzo a finales de noviembre, por lo que para estos educadores era imposible presentarse en Heredia para recibir lecciones durante este período (Aguilar y Angulo, 2007).

Posteriormente, conforme se profundizó en el conocimiento de la realidad rural, el plan de estudios cambia: se le introdujeron mejoras que lo hicieron más pertinente para la realidad rural. Se hicieron ajustes en contenidos; por ejemplo, se incluyó el estudio de la escuela unidocente como fenómeno básicamente rural, la administración escolar, por ser esta una de las tareas del docente en este tipo de escuelas, y las unidades integradas como una forma de abordaje metodológico en las escuelas unidocentes. También, se integraron contenidos en aspectos de salud (primeros auxilios) y recreación (técnicas recreativas para diferentes edades, campamentos), atendiendo a la concepción del maestro como una persona que, a falta de profesionales especializados, apoya los procesos de esta índole en la comunidad. Se incorporó el estudio de la relación con la comunidad, por el papel de liderazgo que los maestros cumplen en la comunidad, por ser, generalmente, las personas con mayor escolaridad.

Se mejoró la metodología, tanto en su fase presencial (mayor duración), como en su fase a distancia, mediante diversas técnicas, guías más elaboradas y una supervisión más cercana.

Otros aspectos que se tomaron en cuenta fueron la inclusión de un criterio de procedencia de las zonas rurales para la selección de estudiantes que cursaran el plan de estudios, y la estabilidad laboral en un lugar de trabajo ubicado en la zona rural de procedencia; ambas, medidas para propiciar el arraigo hacia lo rural.

Muy pronto la DER se dio cuenta de que dentro de lo rural, para el caso costarricense, también se encontraba lo indígena; de manera que incursionó en esta temática, aplicando los mismos principios y metodologías que ya había probado en lo rural. Una vez constatada la necesidad de formación de docentes en esta población, junto a la detección de otras necesidades y características, se procedió a la puesta en marcha de un plan de estudios con cursos como Lengua y comunicación, Metodología para escuelas indígenas, Legislación indígena, Teoría de la cultura y Seminario pedagógico integrativo en la educación indígena, todos ellos pertinentes para el tipo de problemática que vivían estas comunidades (Aguilar y Angulo, 2007).

El trabajo continuó con evaluaciones y revisiones, dio lugar a otros cambios para hacer los ajustes que requería el plan de estudios, según los nuevos conocimientos de la ruralidad y el grado de madurez académica de la División. Así, producto de este trabajo, se abrió una Licenciatura en I y II ciclos con énfasis en Educación Rural, en la que se incluyeron, además de los cursos que ya se habían probado, otros igualmente pertinentes, entre ellos: Administración de la escuela rural, 
Desarrollo comunal, Desarrollo de la creatividad, Introducción a los estudios en la modalidad presencial y a distancia, Educación y Desarrollo Rural, Educación abierta en el contexto rural e Investigación en Educación Rural (Aguilar y Angulo, 2007).

Como la normativa universitaria exige cursos optativos, los que se ofrecieron versaban sobre temáticas de interés para personas que van a laborar en ámbitos rurales: Acercamiento al contexto rural, Organización de bibliotecas comunales, Situación de la mujer en el contexto rural, La escuela unidocente de Costa Rica, Educación agrícola y Educación en prevención de los desastres naturales.

Otros cursos que se ofrecieron en los diversos niveles que imparte la División -Diplomado, Bachillerato o Licenciatura- y que dan muestra del crecimiento de la DER y del seguimiento que dio a la ruralidad para acrecentar su conocimiento del medio, fueron: Educación para la diversidad en la escuela rural, Realidad rural, Educación rural contemporánea, Integración de la educación rural, Pedagogía aplicada en la escuela rural, Ética profesional y docencia rural, Integración curricular en la escuela rural, Educación rural comparada, Diseño de proyectos en las comunidades rurales, Educación ambiental en el contexto rural, Escuela multigrado, Educación no formal en el contexto rural y Educación rural y desarrollo (Aguilar y Angulo, 2007).

La historia de la DER es una historia de crecimiento científico en Pedagogía Rural. Sus avances ofrecen un material muy enriquecedor en lo teórico y en lo práctico. Estos avances son el resultado del trabajo constante de un equipo de académicos que construyó este conocimiento a partir de análisis y discusiones sobre el tema, giras de campo, evaluaciones del trabajo realizado, observación de estudiantes, capacitaciones para docentes de escuelas rurales, formación especializada del personal académico, establecimiento de vínculos estratégicos y algunas investigaciones realizadas en la División.

Cabe mencionar que en este período no se escribió mucho de lo que se hizo, a pesar de que se realizaron una gran cantidad de acciones que contribuyeron a conformar el conocimiento acumulado. Lo anterior, porque la visión de la DER estaba muy orientada a la docencia, y en ello se invertía la mayor parte de los recursos, por lo rudimentario de las facilidades para escribir (máquinas de escribir, reproducción en esténcil, otros), por la ausencia de revistas y otro tipo de medios que ofreciera la posibilidad de publicar trabajos, y por la carencia de incentivos como los que existen en la actualidad para quienes tienen una producción académica escrita (participación en seminarios internacionales, divulgación intra y extra universitaria de los trabajos, entre otros).

\section{HACIENDO PEDAGOGÍA RURAL A PARTIR DE LOS APRENDIZAJES MÁS RECIENTES}

Durante un período de aproximado de diez años, la DER incrementó enormemente su producción académica, sustentada en proyectos de docencia, investigación y extensión, lo mismo que en proyectos integrados. De forma paralela, como consecuencia del aumento en proyectos, la DER fortaleció su presencia nacional e internacional, mediante la participación en diversos eventos, algunos más académicos, otros más políticos, otros más teóricos y otros más prácticos.

Algunos de estos procesos fueron grandes y complejos, otros, en cambio, más sencillos, pero todos igualmente valiosos y con contribuciones importantes sobre las nuevas necesidades de la ruralidad al acercarse el siglo XXI.

Estos trabajos dieron lugar a una nueva forma de hacer Pedagogía Rural, mediante proyectos

que estudiaban procesos, procedimientos, instrumentos y teorías, o atendían, en la práctica, diversos 
aspectos de la realidad y la educación rural. Hubo además un esfuerzo de profesionalización del personal, lo que también ayudó en la construcción de competencias académicas para las tareas que se proyectaban en el futuro.

Con todo esto, la DER sistematizó y amplió sus conocimientos, los divulgó en ámbitos nacionales e internacionales, y se posicionó en uno de los primeros lugares en cuanto a Educación Rural en Latinoamérica. Con ello, y con el esfuerzo sostenido del equipo de académicos, junto a una dirección sólida, comprometida y visionaria, la DER dio un salto cualitativo en sus acciones y se animó a incursionar en terrenos internacionales, en los ámbitos de investigación y posgrado.

Debe destacarse que estas dimensiones son sumamente complejas y sólo pueden realizarse con una gran dosis de persistencia, esfuerzo, creatividad y rigurosidad. Como en todo trabajo de calidad, este ha significado una gran inversión de energía, tiempo y pensamiento, por lo que sus frutos no tardaron en verse. En este caso,se abrieron oportunidades importantes que la DER supo aprovechar y que básicamente provenían de la cooperación holandesa, la cual, con su aporte académico y financiero, acompañó a la DER en esta etapa de su desarrollo y le hizo contribuciones significativas en la construcción de los aprendizajes más recientes.

Con esta madurez, la DER se plantea la necesidad de hacer Pedagogía Rural Centroamericana, lo hace con el desarrollo de una Maestría en Educación Rural Centroamericana y con la investigación de la realidad del istmo, en esta misma temática.

La Maestría cuenta con una serie de características novedosas y muy pertinentes, como lo son la itinerancia por varios países centroamericanos, la semivirtualidad, el diseño por módulos y la composición centroamericana del estudiantado y el profesorado. Los procesos investigativos también han tenido sus virtudes, entre ellas, el proveer de información actualizada, sobre una misma temática, en la región centroamericana, y el haber identificado contactos relevantes para compartir acciones profesionales en la región (Carvajal y Díaz, 2007).

Aunque en estos momentos ya finalizó la primera promoción de la Maestría, lo que hace que exista un cúmulo importante de experiencia, queda mucho por aprender y mucho por consolidar en este programa. Las evaluaciones realizadas, tanto en el proceso como al cierre de la primera promoción, han señalado áreas fuertes que deben mantenerse y áreas débiles que deben superarse. Incluso, ya se han identificado situaciones que deben modificarse a la luz de una segunda promoción, con la finalidad de mejorar el trabajo formativo (Carvajal y Díaz, 2007).

Igualmente, en el área de investigación se han acumulado tanto información pedagógica como logros y limitaciones. Se cuenta con los productos de una investigación en la temática de formación de educadores en varios países de la región centroamericana, lo que permite comparaciones y otras formas de análisis en el plano teórico, y además, permite establecer posibles líneas de colaboración, demanda y oferta de servicios, y participación conjunta en ciertos proyectos, entre otras acciones. También, se avanzó en cuanto a la identificación de profesionales clave en esta temática, investigadoras e investigadores de alto nivel en la región, lo mismo que en la ubicación de fuentes bibliográficas y fuentes de financiamiento para trabajos futuros.

En investigación hay mucho camino que recorrer. Falta una ampliación en los temas de estudio para contribuir con el acervo de conocimiento que da sustento al trabajo regional y falta completar y diferenciar por temas, países y áreas de especialización, la lista de contactos, autores, estudios, instituciones y otros componentes que servirán de insumo y de aliados en el futuro (Carvajal y Díaz, 2007).

En lo inmediato, estas serán las tareas de Pedagogía Rural a las que debe abocarse la DER para consolidar los aprendizajes del momento más reciente, sin descuidar, sino más bien fortale- 
cer, los trabajos que ha realizado en cuanto a formación de educadores en el grado (Bachillerato y Licenciatura) y el desarrollo de trabajo de campo y procesos de capacitación de educadoras y educadores.

En el camino recorrido hay una importante acumulación de conocimientos y experiencias que también constituyen Pedagogía Rural. El interés por temáticas específicas, tales como metodologías, formas de participación, relaciones escuela-comunidad, adecuaciones curriculares y otras, debe remitir a los documentos que se han elaborado para ciertos contextos, con determinados enfoques y que han sistematizado el conocimiento que se produjo en el proceso ${ }^{3}$.

En términos de la construcción de la Pedagogía Rural, estos trabajos han sido los insumos que permitieron el avance hacia un segundo momento, los soportes que dieron lugar a las acciones realizadas y los pilares que sostienen los logros alcanzados.

\section{LA PEDAGOGÍA RURAL DEL FUTURO}

Desde la perspectiva de la Pedagogía Rural, son muchas las tareas que pueden y deben desarrollarse en el futuro. Al respecto, podríamos enumerar temáticas por investigar, situaciones en las que se puede profundizar, acciones que no se pueden postergar y análisis que han estado ausentes en la ruta que tomó la División desde el inicio de sus actividades. Sin embargo, no es interés de este documento entrar en este tipo de reflexión, por cuanto hay situaciones de fondo, en proceso, que tendrán un gran peso en la Pedagogía Rural del futuro ${ }^{4}$.

La selección del camino por tomar depende mucho de todo el trabajo realizado previamente, así como de las decisiones y orientaciones de las nuevas generaciones que conforman actualmente la División, tanto en el profesorado como en el estudiantado. Las y los nuevos profesionales, y las y los nuevos estudiantes, tienen condiciones que van a influir decisivamente en las acciones por tomar, entre estas posiblemente están: ellas y ellos tienen percepciones, visiones y expectativas diferentes a las que regían anteriormente, han incorporado la semilla de los adelantos tecnológicos y han hecho avances importantes en cuanto a la convergencia disciplinaria que debe tener lugar en los procesos académicos y humanos. Todas estas potencialidades ofrecen un terreno fértil para la generación de nuevas ideas y para asumir retos que tal vez ni se sospecharon en las generaciones pasadas.

Otro factor que interviene en el futuro de la Pedagogía Rural, es el cambio social. La dinámica de la sociedad actual influye en todos los procesos y les crea nuevas alternativas, dificultades, orientaciones y conexiones, todo lo cual debe tenerse presente cuando se piense en el camino por seguir. Así, por ejemplo, la globalización, la sociedad del conocimiento y la reciente aprobación de un tratado de libre comercio entre Costa Rica y los Estados Unidos, inevitablemente generan, en la ruralidad, nuevos procesos, condiciones, necesidades, posibilidades y situaciones, que deben considerarse antes de decidir.

El tratado de libre comercio, por sus características y su amplia cobertura en asuntos económicos, educativos, agroindustriales y de otra índole, constituye un hecho, que, por sí sólo, es de gran trascendencia para el futuro de la Pedagogía Rural; sin embargo, sería muy prematuro adelantar

\footnotetext{
El lector podrá encontrar información sobre lo que se ha recomendado para el accionar futuro de la División de Educación Rural en los informes de evaluaciones internas y externas que se han realizado a los diferentes proyectos y planes de estudio de la División, tanto en grado como en posgrado, lo mismo que en los informes de directores y directoras anteriores, y en las actas de los consejos académicos de unidad y de centro.
} 
criterios, por lo incierto de la situación en la actualidad. No obstante, es imprescindible observar cuidadosamente y estudiar las causas, consecuencias e impacto de éste y otros fenómenos en la ruralidad, pues sólo así, la DER podrá mantener su visión actualizada y ofrecer programas pertinentes para Costa Rica y para la región centroamericana.

En estas condiciones, la única perspectiva que con certeza se puede anticipar para la Pedagogía Rural, tiene que ver con mantenerse alerta ante los cambios que pueden darse en el país y en la región, por cuanto esto es determinante para la definición de prioridades y líneas de acción.

Como esta vigilancia sólo puede hacerse con un equipo de trabajo motivado, positivo, comprometido, estudioso de la realidad, sólidamente formado, con deseos de aprender y con voluntad para trabajar en beneficio de la ciencia pedagógica y de los habitantes de las comunidades rurales, resulta siempre pertinente poner atención a la selección, promoción, formación, actualización y estímulo al personal con que se cuenta, todo ello porque en sus hombros descansará el peso de los sacrificios que deban hacerse para crecer y para consolidar el desarrollo de la Pedagogía Rural.

\section{REFERENCIAS}

Aguilar, M. E. y Angulo, L. (2007). Reseña de la División de Educación Rural. Heredia, Costa Rica: Universidad Nacional (DER-CIDE). Aguilar, M. E. y Monge, M. (1994). Hacia una Pedagogía Rural. Costa Rica: UNA-ANDE.

Carvajal, V. y Díaz, A. (2007). Informe de autoevaluación de la Maestría en Educación Rural. Campus Omar Dengo, Heredia, Costa Rica: Sistema de estudios de posgrado. División de Educación Rural.

Universidad Nacional. (2006). Plan de estudios de la Maestría en Educación Rural. Heredia, Costa Rica: División de Educación Rural. 